\title{
PROPOSTA DE UTILIZAÇÃO DE TECNOLOGIAS OPEN SOURCE PARA UM SISTEMA DE GESTÃO DE ADMISSÕES AO ENSINO SUPERIOR
}

\author{
Junior Marimbique e Bráulio Alturas \\ Instituto Universitário de Lisboa (ISCTE-IUL), ISTAR -IUL, \\ Instituto Universitário de Lisboa, Av. Forças Armadas 1649-026 Lisboa, Portugal
}

\begin{abstract}
RESUMO
As instituições de ensino superior são caraterizadas pelo crescimento estudantil, pelo que surge a necessidade de alinhar os seus programas de ensino à demanda do mercado e de adotar novas técnicas de gestão e prestação de serviços, sendo, para o efeito, indispensável o recurso às tecnologias de informação. Este artigo procura desenhar um protótipo para sistema de informação de gestão de admissão ao ensino superior e propor ferramentas de engenharia de software open source que possam auxiliar o seu desenvolvimento. O protótipo foi desenhado seguindo a metodologia de desenvolvimento de software orientada a objetos, e as ferramentas da engenharia de software com base na comparação de duas ferramentas para três elementos de software para sistema de informação (SI) nomeadamente a formulários, relatórios e portal Web.
\end{abstract}

\section{PALAVRAS-CHAVE}

Sistemas de Informação, Tecnologias de Informação, Ensino Superior, Software Open Source

\section{INTRODUÇÃO}

Desde o início do século, têm-se registado transformações na forma de gestão das organizações, motivadas por mudanças internas e externas, entre as quais o crescimento da organização, a globalização e o aparecimento de novas tecnologias. Estas transformações, por sua vez, podem mudar as normas de funcionamento, a estrutura ou os objetivos organizacionais (Alturas, 2013).

As instituições de ensino superior (IES) procuraram alinhar a gestão de processos de acordo com as mais novas premissas da gestão empresarial e da prestação de serviços. Este facto desafiou as IES a alinhar os seus processos de trabalho de acordo com as técnicas de governação empresarial mais modernas, onde os sistemas e tecnologias de informação ocupam um lugar de destaque (Machado, 2008). A utilização de SI, numa instituição de ensino, ajuda a economizar tempo, aumenta a produtividade, permite executar mais tarefas do que de outra forma e, os processos de trabalho sejam menos burocráticos (Chimuco \& Alturas, 2018).

Apesar de inúmeros casos de sucessos do software open source, o software proprietário ainda continua a dominar em boa parte do mercado (Kepes, 2013). Entretanto, este estudo partiu do princípio da existência de diversos softwares open source ou ferramentas da engenharia de software open source aplicáveis no desenvolvimento de SI para ensino superior.

Neste artigo é usada a metodologia de design science research com o objetivo de criar os requisitos funcionais do sistema de admissão de estudantes do ensino superior em uma IES moçambicana, estes requisitos são usados na avaliação da possibilidade de aplicação de software open source. O resultado deste trabalho comprova a existência de software open source reaproveitável para desenvolvimento de SI de ensino superior, com pouco esforço adicional. 


\section{REVISÃO DA LITERATURA}

\subsection{Sistemas de Informação}

Os sistemas de informação contêm dados sobre pessoas, lugares e objetos dentro da organização ou no seu meio envolvente (Laudon \& Laudon, 2018). O eixo comum encontrado na pesquisa do contexto de sistema de informação é de que o mesmo é um conjunto de elementos (Ex: pessoas, dados, processos, tecnologias) interconectados, que visa produzir informações e conhecimento úteis para a organização (Alturas, 2013; Amaral \& Varajão, 2000). Entretanto, Alturas (2013) e Amaral \& Varajão (2000) referem que a existência de sistemas de informação numa organização não depende de computadores ou outros recursos informáticos. $\mathrm{Na}$ realidade, todo o sistema que manipula dados e gera informação, usando ou não recurso a tecnologias da informação, pode ser genericamente considerado como um sistema de informação (Alturas, 2013).

Apesar de os conceitos de sistema de informação acima propostos não referenciarem diretamente a tecnologia de informação, na realidade é cada vez mais raro (...) sistemas de informação sem recursos informáticos (Alturas, 2013). Este pensamento também é defendido por Alter (1999) que adiciona computadores e processos de trabalho organizacional no conceito de sistemas de informação.

\subsection{Sistema de Informação de Ensino Superior}

A busca de eficiência na gestão das IES, fez com que elas alinhassem os seus processos de gestão de acordo com as mais novas premissas da gestão empresarial e da prestação de serviços, onde os sistemas e tecnologias de informação ocupam um lugar de destaque (Machado, 2008). Os fornecedores de tecnologias de informação responderam, inicialmente a massificação do uso de TI no ensino superior, fornecendo produtos adaptados para empresas, devido a similaridade da estrutura administrativa das universidades às grandes empresas (Ahmad et al., 2009).

Entretanto, de acordo com Ahmad et al., (2009) as IES tem a sua natureza única, que o difere das empresas, Lockwood (1985) cita quatro caraterísticas desta unicidade: complexidade dos objetivos, autonomia e dependência da sociedade civil, estrutura e autoridade difusa, limitação na medição de resultados e fragmentação interna.

Outro aspeto não menos importante da diferença entre empresas e IES reside na natureza e orientação destas duas organizações. Enquanto as empresas estão focadas em lucros, as IES são na sua maioria suportadas por fundos públicos e assumem a vocação de ensinar e investigar, sem deixar de lado a necessidade de sustentabilidade (Noaman \& Ahmad, 2015).

As diferenças entre as empresas e as IES estendem-se as funções administrativas, e, por conseguinte, nos sistemas de processamento de transações. Por exemplo, enquanto que o ERP tradicional fornece funções básicas de administração tais como contabilidade, finanças, vendas marketing e recursos humos, os ERP para as IES requerem funções adicionais, que incluem administração de ensino, administração pedagógica, gestão das instalações, gestão de bibliotecas e outras funções que não estão presentes no ERP tradicional. De acordo com Noaman \& Ahmad (2015) a implementação com sucesso de TI nas IES fica condicionado, em primeiro lugar, a definição clara das funções de gestão necessárias.

Outro sistema comumente usado nas IES é o CRM, que apresenta uma visão integrada dos estudantes em todas áreas do negócio, de modo que estes recebam um serviço de alto nível. A satisfação dos estudantes com os serviços de gestão de ensino é um fator importante na avaliação de qualidade de ensino superior, é neste ponto em que o CRM pode desempenhar um papel importante. De acordo com Seeman \& O'Hara (2006) muitos alunos veem os serviços administrativos como um mal necessário. Assim, um SI que aperfeiçoe o CRM, focando-se na redução dos passos das tarefas administrativas, pode tornar a IES mais competitiva.

O Avanço das tecnologias e a popularização internet no seio da população mundial, tem permitido a massificação uso do e-learnig nas IES, equiparando-se a sistema para e-business nas empresas. Sistemas de e-learning englobam o conjunto de tecnologias e metodologias para desenhar, disponibilizar, administrar, selecionar e estender os serviços da educação a pessoas distantes do espaço físico da IES. O conceito ideal do e-learning, não se resume a transposição de conteúdos ministrados na presencialmente na plataforma, o e-learning deve envolver todos outros serviços prestados ao estudante normalmente no campus, que inclui, o empréstimo de livros, acesso ao e-book, serviços de gestão de ensino, tesouraria, apoio ao estudante, dentre outros. 


\subsection{Software de Código Aberto}

\subsubsection{Tecnologias Open Source}

Nos últimos anos, são cada vez mais os casos de sucesso que se registam na indústria do software open source, motivando engenheiros de softwares e homens de negócio a adotarem o software de código aberto em seus projetos. Um software open source é aquele que pode ser distribuído gratuitamente, seu código disponibilizado sem custos e com permissão de alterar e redistribuir o software sob certos termos de licença (Liaskos et al., 2012). Os termos de licença dos softwares open source têm em vista proteger os direitos autorais, estabelecer os termos de distribuição dos produtos derivados e manter o fluxo do software de código aberto (Kon et al., 2011).

Os benefícios do software open source comumente aceites estão agrupados em cinco categorias: benefícios sociais e psicológicos, benefícios da metodologia de desenvolvimento do Software, benefícios de segurança e gestão de riscos, benefícios da adoção do software ao longo a vida e benefícios financeiros (Rooij, 2014).

A simples obtenção de Software de código aberto não significa que se possa fazer o que quiser com ele. A licença open source é o documento através do qual os detentores de direitos autorizam o uso das suas obras e as respetivas restrições. Cada software open source pode ter a sua licença especifica, porém, a prática comum é reaproveitar as licenças já consolidadas no seio da comunidade. Outro fator a ter em conta na licença do software open source é a compatibilidade entre licenças, uma vez que algumas soluções tecnológicas recorrem a combinação de códigos de dois ou mais softwares, que podem ter licenças diferentes. A recomendação neste caso é analisar os potencias conflitos de licença antes de iniciar um projeto de reuso de software.

\subsubsection{Tecnologias Open Source nas Instituições de Ensino Superior}

A operacionalização das IES pode ser racionalizada reduzindo os custos com as tecnologias de informação. O uso de aplicações open source para a gestão de conteúdos e gestão académica tem sido uma das formas para racionalizar os recursos nas IES (Rooij, 2014), visto que com software open source, os desenvolvedores podem personalizar o sistema de modo a atender as necessidades das IES, reduzindo os custos com licenças comerciais, principalmente as de pagamento periódico.

A oferta de tecnologias open source ocupa hoje um lugar de destaque no ensino superior, na medida que oferecem um conjunto de requisitos que respondem por completo às necessidades de grande parte das organizações que procuram redefinir seus processos de trabalho baseando-se nas tecnologias de informação (Ávila, Teixeira, \& Almeida, 2012).

De acordo com Rooij (2009), a oferta de tecnologias open source para educação, não se limita apenas ao sistema de gestão de conteúdos ou a ambientes virtuais de aprendizagem. Estes podem incluir navegadores de internet, software de transferência de dados (encriptação), software de gestão de redes, software de roteamento, sistemas operativos, servidores aplicacionais e até sistemas de gestão financeira.

Os principais fatores que contribuem para a adoção do software open source no ensino superior são a necessidade de racionalização de fundos, o aumento do preço das licenças de uso de software comercial e a necessidade das IES adquirirem cada vez mais licenças de software (Rooij, 2009). Estes três fatores enfatizam a influência dos benefícios financeiros na adoção das tecnologias open source no setor do ensino superior.

\section{METODOLOGIA}

Neste trabalho foi usada a metodologia de design science research (DSR), visto que a intenção é de construir um artefacto, e embora se possa utilizar outra metodologia para construir um artefacto, esta foi considerada a mais adequada. DSR é a metodologia de pesquisa para a construção de qualquer artefacto como solução incorporada para um problema de pesquisa conhecido e compreendido (Peffers et al., 2007), ele envolve um processo rigoroso para projetar artefactos, resolver problemas observados, fazer contribuições de pesquisa, avaliar modelos e publicar os resultados (Hevner et al., 2004). Com vista a alcançar os objetivos propostos, o 
trabalho compreendeu a análise dos utilizadores, análise de requisitos e avaliação de software open source aplicável para a gestão do processo de acesso de estudantes ao ensino superior.

A avaliação dos stakeholders e suas necessidades dos foi efetuada por meio de entrevista, em que se esperam encontrar as caraterísticas das diferentes classes de utilizadores, bem como os seus objetivos. A entrevista foi dirigida a pessoas mais envolvidas na administração de acesso a IES e a estudantes e/ou candidatos a estudantes com vista a encontrar as suas necessidades.

A avaliação do software open source compara os requisitos do sistema às funções ou propriedades do software. Pare este efeito foram selecionados seis software open source sendo dois de produção de relatórios, dois de produção gestão de formulários e dois para o desenvolvimento de portais Web. A comparação da Software centrou-se nas funcionalidades, versatilidade, dinâmica da comunidade de desenvolvimento e modelo de negócio.

\section{ANÁLISE E DESENHO DO SISTEMA}

A análise dos intervenientes do sistema permite identificar as características das pessoas que utilizam o sistema e seus objetivos. A entrevista a estudantes, candidatos e membros da comissão de exames de admissão do ISPM, os processos, o relacionamento entre processos e os stakeholders, bem como os seus objetivos. Assim, foram entrevistados 5 candidatos, 5 estudantes e 10 membros da comissão.

A entrevista aos membros da comissão de exames de admissão do ISPM permitiu-nos constatar que as principais classes de stakeholders do sistema são: candidatos, IES e instituições governamentais gestoras do ensino superior. A análise dos objetivos centrou-se nos candidatos e nas IES, visto que esses são atores de ação direta do sistema.

O cadastro é o primeiro passo, para dar início ao processo de candidatura. Nele se estabelecem os mecanismos de autenticação na plataforma e mecanismos de comunicação entre os utilizadores. $\mathrm{O}$ email e telemóvel são as formas mais utilizadas para comunicação eletrónica em Moçambique, pelo que se propõe que o sistema utilize a autenticação por email ou a autenticação por telemóvel.

$\mathrm{Na}$ apresentação da candidatura, devem-se submeter os dados pessoais do candidato e a documentação exigida, podendo estes serem subdivididos em obrigatórios e opcionais.

A análise da candidatura consiste em verificar a conformidade da candidatura, efetua-se a avaliação de dados e documentação com os requisitos exigidos. Após este processo é anunciado o estado da candidatura, podendo ser aceite ou não aceite.

A avaliação da candidatura pode ser por análise de documentos ou exames. Na admissão por avaliação documental, a documentação a ser classificada é atribuída uma pontuação e a média ponderada da pontuação dos documentos classificáveis serve de classificação final dos candidatos. $\mathrm{Na}$ avaliação por exame os candidatos são submetidos a um ou mais exames e tal como na avaliação documental a classificação final é a média ponderada das pontuações dos exames.

\section{AVALIAÇÃO DE SOFTWARE DE CÓDIGO ABERTO}

Avaliar consiste em comparar uma determinada realidade a um modelo ideal, designado de padrão. Neste processo efetuou-se a comparação de duas ferramentas aos requisitos do sistema. Os componentes necessários para o sistema eletrónico de gestão de admissão são essencialmente gestor de formulário, ferramenta de produção de relatório e portal web. Sendo a avaliação de software centra-se na potencialidade destas ferramentas no desenvolvimento do sistema.

\subsection{Gestor de Formulários}

Os formulários constituem um essencial elemento para a comunicação com os utilizadores do sistema, e um dos elementos essenciais para a introdução de dados em qualquer sistema de informação. $\mathrm{O}$ gestor de formulário, neste contexto, tem a responsabilidade de criar, formatar, alterar e remover campos de introdução de dados de acordo com a informação a receber. 


\subsubsection{Descrição das Ferramentas}

WebForm é uma ferramenta de gestão de formulários no sistema de gestão de conteúdos (SGC) Drupal. O WebForm permite a criação, submissão, revisão, obtenção de dados e envio de notificações. O WebForm é usado em 480000 sites e está sob a mesma licença do Drupal, GNU General Public License. A versão 5.x do webform foi completamente reimplementada como código fonte do Drupal 8, aperfeiçoando a sua integração.

Orbeon Forms CE é uma ferramenta de gestão de formulários, que permite a criação e publicação de formulários web que inclui um mecanismo XForms, o construtor de formulários e o editor de formulários. Esta ferramenta possui a versão de código fechado e a versão Community open source, com o código disponibilizado no github sob a GNU Lesser General Public License. Ela é desenvolvida em Java pela Orbeon, Inc.

\subsubsection{Relatório de Comparação}

As duas ferramentas de gestão de relatórios foram instaladas de forma a comparar as suas funcionalidades e verificar a seu alinhamento com os requisitos para o sistema de gestão de acessos ao ensino superior. Verificou-se ser mais fácil instalar e configurar o Drupal + Webform relativamente ao Orbeon Forms CE, entretanto, o Orbeon Form dispõe de uma interface mais agradável.

O facto de do webform não suportar a o campo de entrada de resposta ( $\mathrm{Sim} / \mathrm{Não}$ ) é desconsiderado por ser possível conseguir mesmos efeitos no campo de seleção. Ambas as ferramentas dispõem de mecanismos de validação de dados de entrada, com exceção à validação de arquivos, que necessita de adição de código para o efeito.

Com relação aos mecanismos de gestão de formulários e submissão, regista-se a comodidade nas duas ferramentas, ambas fornecem os mesmos mecanismos de gestão.

Tanto o Webform assim com Orbeon disponibilizam a documentação oficial que incluem instruções para instalar, construir, publicar e gerir formulários. O Webform, para além da documentação oficial, dispõe também da documentação da comunidade de desenvolvedores que aborda a estrutura da software e a API, assuntos diminutamente abordado na documentação do Orbeon.

\subsection{Ferramentas de Produção de Relatórios}

\subsubsection{Descrição das Ferramentas}

BIRT, acrónimo para Business Intelligence and Reporting Tools, é uma ferramenta open source, para visualização de dados e criação de relatórios. O BIRT é um componente do Integrated Development Environment (IDE) Eclipse que cria uma perspetiva para desenvolvimento de relatórios e gráficos. O BIRT está disponível sob a Eclipse Public License.

O BIRT tem recursos para a criação de relatórios, gráficos complexos suportando uma vasta gama de fontes de dados, entre elas o POJO data source, NoSQL, JDBC, Scripted database. No que diz respeito a formatos de relatório é possível extrair em PDF, Spreadsheet e XLS.

O JasperSoftStudio permite a criação de templates, expressões complexas e diversos componentes de relatórios que incluem tabelas, tabelas de referências cruzadas, listas e gráficos. No que diz respeito a fontes de dados, ela suporta o big data, JDBC, NoSQL, CSV, JSON, XML, JavaBeans ou ainda fontes de dados personalizadas. A publicação dos relatórios desenvolvidos pela jaspersoft studio pode ser feita por meio do jaspersoft server, ferramenta também com a edição open source disponível. Para este efeito, existe um mecanismo técnico de ligação entre as duas ferramentas, que possibilita a publicação de relatórios. Tanto jaspersoft Studio, como jaspersoft server estão disponíveis sob a licença Affero General Public License e são mantidas essencialmente pela TIBCO Software Inc.

\subsubsection{Relatório de Comparação}

As duas ferramentas de produção de relatórios foram instaladas de forma a comparar as suas funcionalidades e verificar o seu alinhamento com os requisitos para o sistema de gestão de acessos ao ensino superior. Verificou-se uma tendência de comodidade aplicacional nas duas ferramentas. As diferenças concentram-se essencialmente no modelo de negócio. 
Tanto BIRT assim como o Jaspersoft studio suportam o SQL, NoSQL, XML, CSV e objetos java como fontes de dados. Esta vasta gama de fontes de dados suportadas é em parte justificada pelo facto de as duas ferramentas usarem o JDBC, recurso do Java para se conectarem a fontes de dados. A notória diferença está nos tipos de objetos Java obtidos suportadas como fonte de dados, sendo que o BIRT suporta os Plain Old Java Objects (POJO) enquanto que JasperSoft Studio privilegia o JavaBeans.

No que diz respeito aos itens de produção de relatórios, verifica-se que ambas as ferramentas dispõem dos mesmos itens de produção de relatórios, verificando-se apenas alguma diferença nos tipos de gráficos disponíveis, mas que de acordo com os requisitos do sistema de admissão ao ensino superior, consideram-se ambas ferramentas compatíveis no que diz respeito aos itens de desenho de relatórios e templates.

O mecanismo tradicional de apresentação dos relatórios é em forma de página web, sendo exportáveis para outros formatos. O BIRT pode ser obtido ou exportado para mais formatos de documentos em comparação com o JasperSoft Studio. Entretanto, outros formatos de documentos de relatórios podem ser gerados ou exportados de ambas as ferramentas, requerendo desenvolvimento adicional para este efeito. Tanto o BIRT, como o JasperSoft Studio não dispõem de interface gráfica para geração de queries, ambas apresentam mecanismos simplificados para a criação de queries SQL.

De acordo com a Tabela 1, que procura medir a dinâmica da comunidade open source nas ferramentas em análise, o BIRT tem a comunidade de desenvolvimento mais dinâmica, uma vez que conta com a participação de três empresas de topo no ramo de tecnologias, para além da maior intervenção da comunidade no processo de desenvolvimento e gestão do produto.

\subsection{Portal Web}

Um Portal Web é um sistema desenhado para prover informações de diversas fontes. Com a possibilidade de o sistema de admissão ao ensino superior ser constituído por diferentes ferramentas, aliado ao facto de ser necessário disponibilizar aos stakeholders outras informações da IES, o portal web assume um papel preponderante.

Muitas organizações tem adotado o sistema de gestão de conteúdos (SGC) como ferramenta de gestão de portal web, agregando toda informação necessária na comunicação com o publico de forma rápida (Rainville-Pitt \& D’Amour, 2009). SGC são softwares para criação rápida e fácil de websites, em que pessoas sem o conhecimento da programação podem criar, publicar e atualizar conteúdos. Por outro lado, o SGC oferece ao designer e desenvolvedores mais flexibilidade na personalização da aparência e funcionalidades do site (Ghorecha \& Bhatt, 2013). Os sistemas de gestão de conteúdos (SGC), apesar de parecerem virados à facilidade de introdução de conteúdos Web sem a necessidade do conhecimento de linguagens de programação, oferecem também frameworks poderosas que facilitam a comunicação com outros sistemas, constituindo-se assim numa importante ferramenta para o desenvolvimento de portais Web. Neste trabalho foi analisado dois populares SGC open source, o Joomla e Drupal.

\subsubsection{Descrição das Ferramentas}

O Joomla é um SGC que se centra na facilidade de criar, editar, publicar e arquivar conteúdos Web. A capacidade de processar dados proveniente de outras fontes de dados é graças ao seu framework, que tem mecanismos simplificados de adicionar add-ons que estendem o poder deste SGC, por este motivo o Joomla pode ser considerado tanto como um produto para o utilizador final, assim como uma ferramenta da engenharia de Software. Seu uso varia de simples blogs a portais poderosos.

O Drupal procura distanciar-se dos outros SGC, ao dar foco na segurança, standards de desenvolvimento e framework, estes fatores justificam o seu uso como portal de grandes empresas e em situações em que existam múltiplas fontes de dados. O framework do Drupal, muitas vezes citado como poderoso e escalável, tem a capacidade de integração com outros serviços e aplicações, sendo esta também caraterística o elemento principal de marketing da ferramenta. Ao contrário do Joomla, o Drupal é mais considerado como ferramenta de engenharia de Software que um SGC. 


\subsubsection{Relatório de Comparação}

Os mais famosos sistemas de gestão de conteúdos desenvolvidos em PHP apresentam tendência de comodidade funcional, isto faz com que as comparações entre estas ferramentas se centrem em aspetos não funcionais tais como escalabilidade, velocidade, versatilidade, API e dinâmica da comunidade de desenvolvimento.

O Drupal tem suporte a mais sistemas de base e dados e servidores Web, sendo que ambos não suportam bases de dados não relacional. O facto do Joomla não suportar SGBD populares como o MariaDB e SQL Lite deixa-o em desvantagem competitiva neste aspeto.

O facto do Joomla não suportar o Hiawatha, pode não ter grande impacto devido à baixa cota de Mercado deste Web server, mas isto posiciona o Drupal como mais versátil que o Joomla.

Tabela 1. Dinâmica da comunidade de desenvolvimento

\begin{tabular}{|l|c|c|c|c|c|c|}
\hline & \multicolumn{2}{|c|}{ Gesto de formulários } & \multicolumn{2}{c|}{ Ferramenta de relatórios } & \multicolumn{2}{c|}{ Portal web } \\
\cline { 2 - 7 } & WebForm & Orbeon & BIRT & JasperSoft Studio & Joomla & Drupal \\
\hline Fórum de debate & 3 & 1 & 3 & 2 & 3 & 3 \\
\hline Fórum de dúvidas & 3 & 1 & 3 & 3 & 3 & 3 \\
\hline Perguntas frequentes & 3 & 2 & 3 & 1 & 3 & 3 \\
\hline Help Desk dedicado & 3 & 3 & 3 & 2 & 3 & 3 \\
\hline Gestão de incidentes & 3 & 1 & 3 & 1 & 3 \\
\hline $\begin{array}{l}\text { Outras entidades participantes } \\
\text { no desenvolvimento }\end{array}$ & 3 & 0 & 3 & 0 & 3 \\
\hline $\begin{array}{l}\text { Documentação para } \\
\text { desenvolvedor }\end{array}$ & 3 & 2 & 3 & 2 & 3 \\
\hline $\begin{array}{l}\text { Documentação para uso do } \\
\text { aplicativo }\end{array}$ & 3 & 3 & 3 & 3 & 3 & 3 \\
\hline Centro de desenvolvimento & 3 & 3 & 3 & 3 & 3 & 3 \\
\hline $\begin{array}{l}\text { Proposta de solução de bugs } \\
\text { pela comunidade }\end{array}$ & 3 & 2 & 3 & 3 & 3 & 3 \\
\hline $\begin{array}{l}\text { Adição de novos módulos/ } \\
\text { Funcionalidades pela } \\
\text { comunidade }\end{array}$ & 3 & 1 & 3 & 3 & 3 & 3 \\
\hline $\begin{array}{l}\text { Melhoria de código } \\
\text { SOMA }\end{array}$ & $\mathbf{3 6}$ & $\mathbf{1 9}$ & $\mathbf{3 6}$ & & 3 \\
\hline
\end{tabular}

Apesar dos dados da Tabela 1 sugerirem o mesmo nível da dinâmica da comunidade de desenvolvimento, importa referir que o Drupal tem uma comunidade de desenvolvedores constituído por mais programadores em relação ao Joomla, e fornece melhor suporte de documentação.

\section{CONCLUSÃO}

Este artigo que partiu do princípio da existência de tecnologias open source para o sistema de gestão de admissão a IES, para o efeito, apoiou-se da design science research methodology obter os principais requisitos do sistema bem como o desenho de diagramas e templates de relatórios.

Durante a análise e desenho do sistema, verificou-se que a maior parte dos objetivos dos candidatos estão relacionados com a disponibilidade de informações relacionado com os cursos, IES e local de estudos, para este efeito, o portal web e as redes sociais constituem os principais meios de interação. O cruzamento dos objetivos da IES e dos candidatos com elementos de ação do SI permitiu-nos identificar os elementos de entrada, reporter e difusão de dados ou informação mais críticos para sistema de admissão ao ensino superior. 
Isto fez com que o a avaliação de software open source se centrasse nas ferramentas para gestão de formulários, ferramentas de produção de relatórios e sistema de gestão de conteúdos.

A avaliação do software concluiu que quanto mais liberdade a licença conceder, mais dinâmica é a comunidade do software e quanto mais dinâmica a comunidade for menos riscos para a adoção. Este artigo propõe a adoção do drupal, webform, e BIRT como ferramentas para o desenvolvimento do SI do caso de estudo, sendo que a aplicação em outras IES carece de mais análises.

Os objetivos deste trabalho foram atingidos através da obtenção dos requisitos funcionais e proposta de componentes de software para o desenvolvimento. Entretanto este trabalho está limitado por um lado, a dados colhidos em uma única IES, por outro lado, não aborda a análise de dados, uma componente essencial na produção de informações para tomada de decisões. Recomenda-se mais estudos, envolvendo mais IES, comparação de processos de admissão a IES em países diferentes, comparação IES pública versus IES privada, comparação dos requisitos de estudantes com os dos funcionários e a componente de análise de dados de admissão ao ensino superior.

\section{REFERÊNCIAS}

Ahmad, R., Wasana, B., \& Guy, G. (2009). ERP systems in the higher education sector: A descriptive case study. Proceedings of the Twentieth Australasian Conference on Information Systems., 456-470.

Alter, S. (1999). A GENERAL, YET USEFUL THEORY OF INFORMATION SYSTEMS. Communication of Association of Information System, 1(13).

Alturas, B. (2013). Introdução aos sistemas de informação organizacionais (1.a ed.). Silabo.

Amaral, L., \& Varajão, J. (2000). Planeamento de sistemas de informação. FCA - Editora de Informática, Lisboa.

Ávila, L., Texeira, L., \& Almeida, P. (2012). Desmaterialização de processos com recurso a tecnologias open-source numa instituição de ensino superior. Conferência da Associação Portuguesa de Si stemas de Informação, 12, 200-211.

Chimuco, Y., \& Alturas, B. (2018). Avaliação do impacto do Hipermanager nos processos de trabalho: Aplicação aos docentes de dois colégios em Moçâmedes, Angola. $18^{\text {a }}$ CAPSI Conferência da Associação Portuguesa de Sistemas de Informação, Santarém, Portugal, 5.

Falkenberg, E., Hesse, W., Lindgreen, P., Nilsson, B., Oei, J., Rolland, C., Stamper, R., Assche, F., Verrijn-Stuart, A., \& Voss, K. (1998). FRISCO - A Framework of Information System Concepts. ftp://ftp.leidenuniv.nl/pub/rul/frifull.zip

Hevner, V., March, S., Park, J., \& Ram, S. (2004). Design Science in Information Systems Research.

Kepes, B. (2013). Open Source Is Good and All, But Proprietary Is Still Winning. Forbes.

Kon, F., Labo, N., Meirelles, P., \& Sabino, V. (2011). Software Livre e Propriedade Intelectual: AspectosJurídicos, Licenças e Modelos de Negócio. Congresso da Sociedade Brasileira da Computacao, 59-104.

Laudon, K., \& Laudon, J. (2018). Management Information System: Manage the Digital Firm (15.a ed.). Pearson.

Liaskos, S., Khan, S. M., Litoiu, M., Jungblut, M. D., Rogozhkin, V., \& Mylopoulos, J. (2012). Behavioral adaptation of information systems through goal models. ScienceDirect, 37, 767-783.

Lockwood, G. (1985). Universities: The management challenge. Society for Research into Higher Education.

Machado, L. (2008). Gestão estratégica para instituições de ensino superior privadas.

Morenet. (2019a). Instituições Conectadas a Morenet. http://www.morenet.ac.mz/index.php/pt/sobre-nos

Morenet. (2019b). Servicos da Morenet. http://www.morenet.ac.mz/index.php/pt/sobre-nos

Noaman, A., \& Ahmad, F. (2015). ERP Systems Functionalities in Higher Education. Procedia Computer Science, 65.

Peffers, K., Tuunanen, T., Rothenberg, M., \& Chatterjee, S. (2007). A Design Science Research Methodology for Information Systems Research. Journal of Management Information Systems, 24, 45-77.

Rooij, S. (2009). Adopting Open Source Software Applications in US Higher Education a Cross Disciplinary Review of the Literature. Review of Education Research, 79(2), 682-701.

Rooij, S. (2014). Perception of Open Source Versus Commercial Software. Jornal of Research on Techology in Education, 39(4), 433-453.

Salimo, G., \& Gouveia, L. (2016). Ensino Superior em Moçambique. Os desafios da gestão na Era Digital. Universidade Fernando Pessoa.

Seeman, E., \& O'Hara, M. (2006). Customer relationship management in higher education Using information systems to improve the student-school relationship. Campus-Wide Information Systems, 23, 24-34. 